\title{
Analyses of the robustness of the mathematical model with the laboratory prototype of the drilling process during simulation and vibration control experiments
}

\author{
F. Abdul Majeed ${ }^{1}$, H. Karki ${ }^{1}$, Y. Abdel Magid ${ }^{2} \&$ M. Karkoub ${ }^{3}$ \\ ${ }^{I}$ Department of Mechanical Engineering, \\ The Petroleum Institute, U.A.E. \\ ${ }^{2}$ Department of Electrical Engineering, The Petroleum Institute, U.A.E. \\ ${ }^{3}$ Mechanical engineering Department, Texas A\&M University, Qatar
}

\begin{abstract}
The drilling industry is faced with many challenges, and the sudden failure of a drill string during drilling is one of major concern. Exploration of the causes for the failures reveals vibrations as the major cause. In order to test and analyze the vibration patterns of rotary drilling, a laboratory proto type of the process is set up. The mathematical model developed to analyze the vibration presents residual error. Robustness issues pertaining to model error and modelling error is discussed. Methods to counter the errors and minimize the vibrations are also discussed.
\end{abstract}

Keywords: rotary drilling, robustness, modeling error, vibration, experimental set up, unbalanced mass, parameter uncertainty.

\section{Introduction and problem formulation}

There are many types and designs of drilling rigs. Drilling rigs are classified by the power used to drive the process (electric, mechanic or hydraulic), height of the derrick or the type of drill pipe used (cable, conventional and coil tubing).

The drilling rig we are concentrating on is an electric driven, conventional rotary drilling, fig. 1 . This is the widely used method of drilling and drilling is achieved by the drill bit as it rotates and cuts into rock. All the major items of machinery used in the rig are driven by electric motors. Metal or plastic drill 


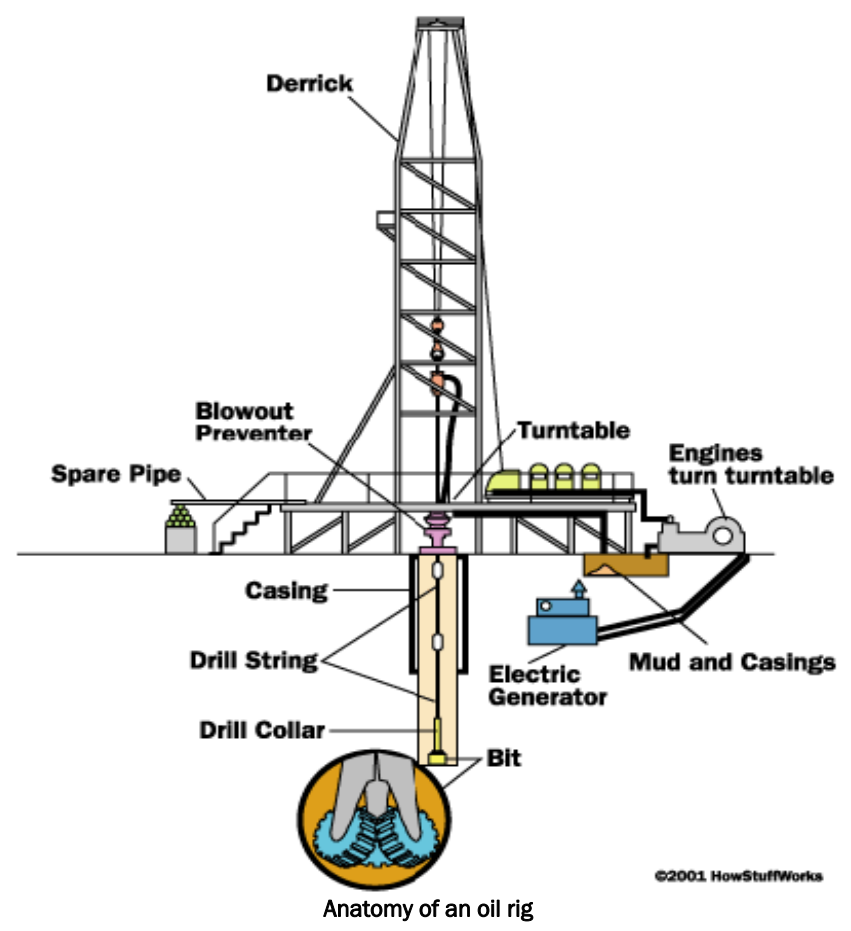

Figure 1: $\quad$ Rotary oil rig features.

pipe for the length of the drill string, and the rotation is achieved by turning a square or hexagonal pipe (the "Kelly") on a rotary table at drill floor level.

Before drilling, a large, heavy bit is attached to the end of a hollow drill pipe. As drilling progresses, the drill bit forces its way underground and additional sections of pipe are connected at the top of the hole. The derrick is the name for the structure which supports the rig above the surface. The taller the derrick, the longer the sections of drill pipe that the derrick can hold at a time. Although early derricks were made of wood, modern derricks are constructed of highstrength steel. Throughout the rotary drilling process, a stream of fluid called drilling mud is continuously forced to the bottom of the hole, through the bit, and back up to the surface. This special mud, which contains clay and chemicals mixed with water, lubricates the bit and keeps it from getting too hot. It also acts as a cap to keep the oil from gushing up.

The drill strings experience high vibrations and strong rocks in the path of drilling. These cause the dynamics presented by drill strings to be highly complex, non linear and unexpected. The drill string vibrations coupled together with well bore friction result in many phenomenon such as bit bounce, stick slip, forward and backward whirl. There are three main types of drill string vibration:

Axial vibration is mainly caused when drilling with roller cone bits. It leads to jumps in drilling; bouncing and can slow down the rate of penetration. 
Torsional vibration results due to twisting of the drill string, and sometimes breaking. It makes the rotation of the drill bit irregular and leads to the stick slip phenomenon.

Lateral vibration occurs when the drill string is bent or when drilling in a non vertical well. The drill bit rotates with a center of rotation not coincident with the center of well, leading to hole enlargement and forward or backward whirl of the bit.

This research concentrates on lateral vibrations occurring in the drill pipe due to a bend. Ideally with zero well bore friction and assuming the drill string is a perfect straight beam rotated with an axial load, there will be no nonlinearities or vibrations during drilling. However, in the presence of curved/inclined boreholes or unbalanced WOBs the friction between the drill bit and well borehole contact is uneven and different at different contact points. This result in the drill bit centerline not being in the center of the hole, hence the centrifugal force will now act as the center of gravity causing the drill string to bend. Bend drill strings do not follow circular trajectories, causing the drill bit to hit the sides of the borehole. This will eventually lead to the stick slip phenomenon, in which large vibrations and sudden unexpected drill bit movements occur. The usual solution in oil rigs is to stall the entire drilling process, and restart. In extreme cases the drill string would break requiring a call for an entire process up haul.

\section{Experimental set up and literature review}

In order to understand and analyze the vibrations due to the bend drill string, experiments are conducted. The drilling process is simulated in the laboratory by a simple experimental set up, fig. 2. The set up operates by operating a motor, which rotates a large rotor connected to the motor shaft. A drill string (made of carbon steel chosen due to its proximity in properties to actual drill string material) is attached to the upper rotor by a universal joint. This joint provides two degree of rotational freedom ( $\mathrm{x}$ and $\mathrm{y}$ axes). The motor rotation provides the set up with $1 \mathrm{DOF}$ of rotation about the $\mathrm{z}$ axes. In order to understand the behavior pattern of a bend drill string and accurately simulate its trajectories, a literature review is conducted. The following are the literature review results required for accurate modeling of the bend drill string features.

The performance of drill strings and their effect on drilling performance have been investigated and analyzed in a number of researches [1-8]. Many different models were set up to analyze drill string vibrations including lateral vibrations (whirl) and mode coupling [3, 9, 10]. Other researchers have focused on models which represent torsional vibration and have attempted to suggest methods to avoid stick-slip behaviour [2, 7]. Jansen [11] proposed an unbalanced mass model to represent a bend drill string section in which self excited vibrations were studied. Similar models were also studied by other researchers; for example, Melakhessou et al. [5] modelled the drill string as an unbalanced rotor supported by two bearings and research is concentrated on a contact zone between the drill string and the borehole wall. Dykstra et al. [1] explains that the 


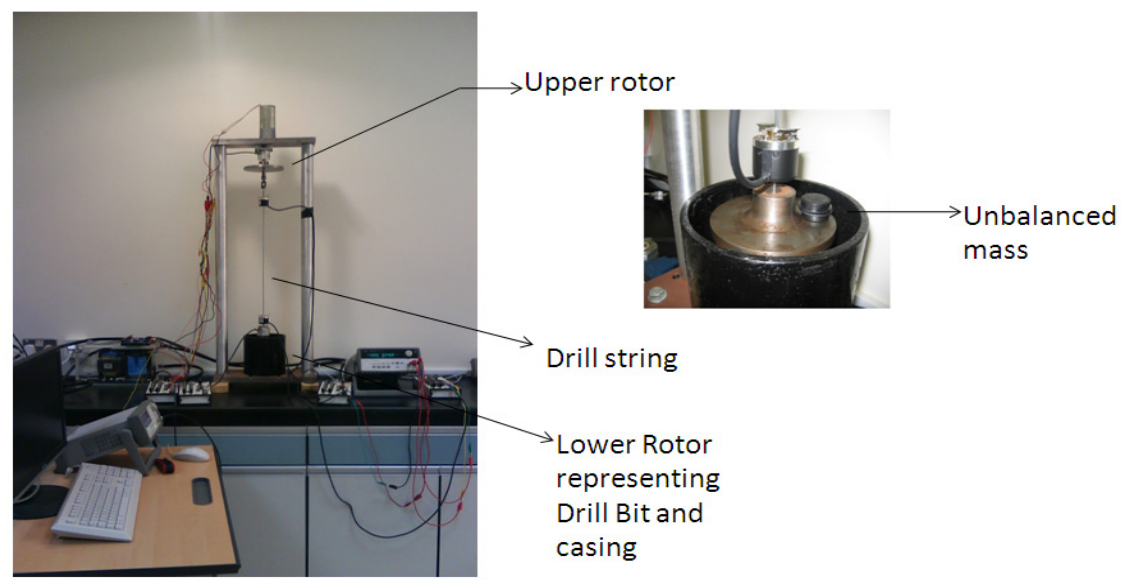

Figure 2: Laboratory drilling set up and close up of lower bit with unbalanced mass.

source of vibration is the bit and hence the centrifugal forces developed when an unbalanced drill string is rotated can be one of the major sources of vibrations.

Analyzing the literature, an unbalanced mass is placed on the lower rotor representing the drill bit to simulate the bend drill string properties. The experimental set up now has three DOFS. Apart from the rotation of the upper rotor, and lower rotor, there is tangential angular displacement for the lower rotor initiated by the new centre of rotation of the lower rotor not coinciding with the centre of rotation of the upper rotor. The lower rotor now follows an elliptical trajectory, also known popularly as bit whirl in the drilling field. This paper also analyses the behaviour of the system at low and average operating speeds of actual drilling.

\section{Robustness issues}

\subsection{Residual error and Model error}

The mathematical model for the process was identified using the system identification black box modeling approach. The experimental set up was excited with chirp input to obtain the required identification data. The chirp input has correlation function properties very similar to white noise. A Box Jenkins model was identified for the process. Box Jenkins models are especially useful when the process is affected by disturbances entering late into the system.

The laboratory operating speeds are selected to represent the rotary drilling process at its low and average operational speeds. The process is excited by command inputs of 8 RPM and 38 RPM, figs. 3 and 4. The usual rotary drilling speeds are around 35 to 45 RPM. The low speed is analyzed to understand the drill string behavior in the transient time. The upper rotary speed is the input speed of the process. The experimental responses of the process are recorded and 
plotted in figures 3 and 4 . The identified model is seen to have very close response to the process response. The residual signal graph between the process response and model response is seen to be very low with values around $180 \mathrm{~m}$ RPM, figs. 5 and 6 . This suggests that the model provides a good fit for analyzing the process behavior.

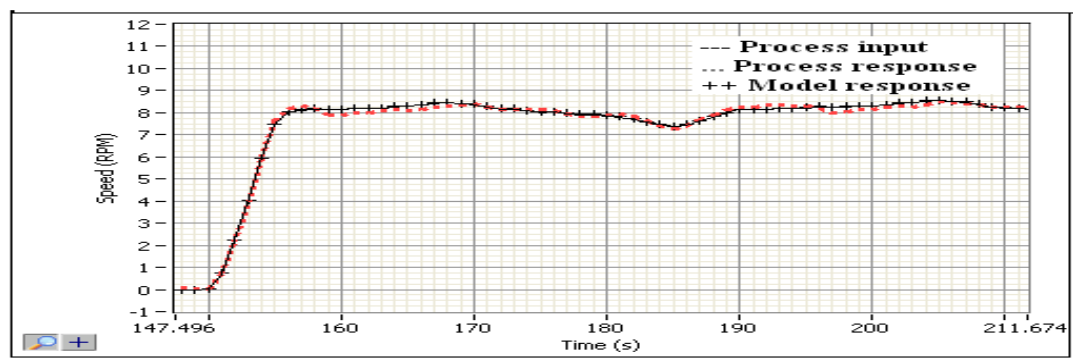

Figure 3: $\quad$ Process data and model response for low drilling speed.

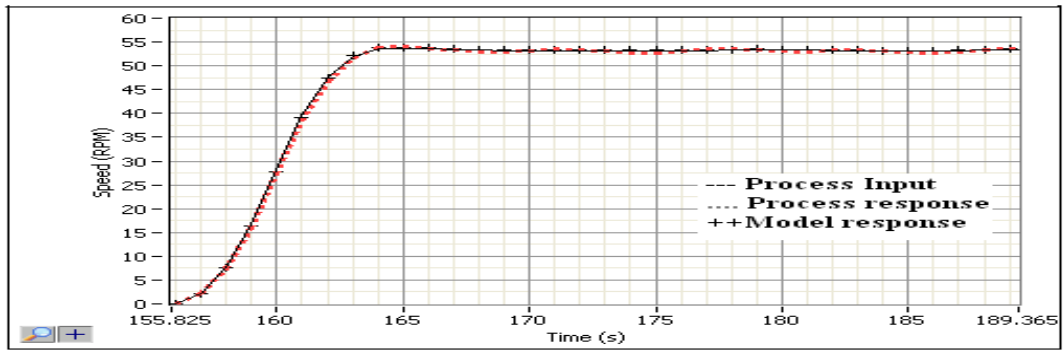

Figure 4: $\quad$ Process data and Model response at average drilling speed.

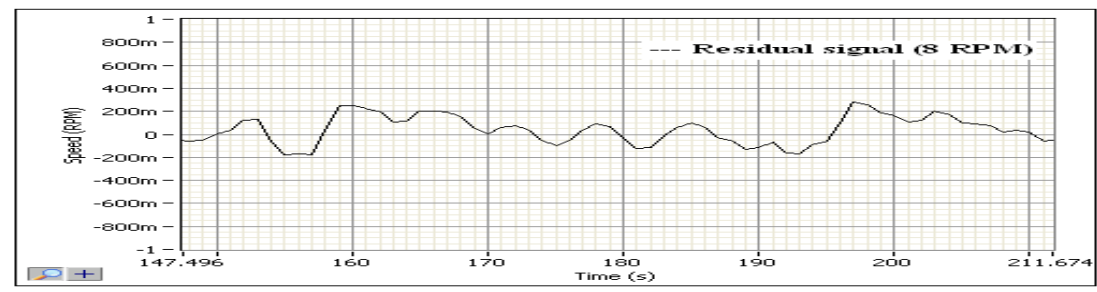

Figure 5: Residual signal for low speed analysis.

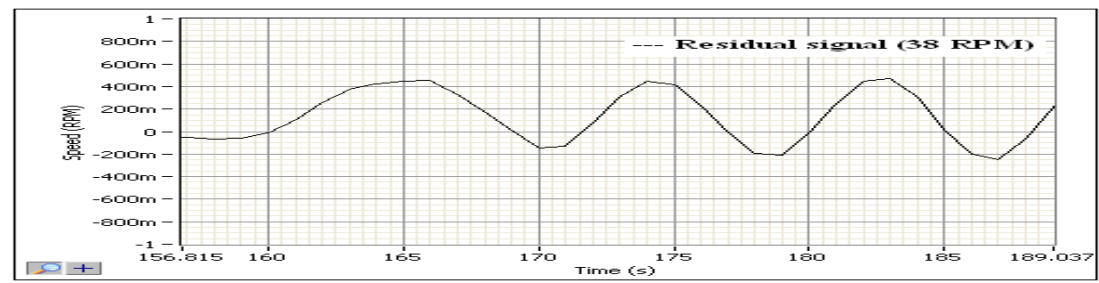

Figure 6: Residual signal for average speed analysis. 


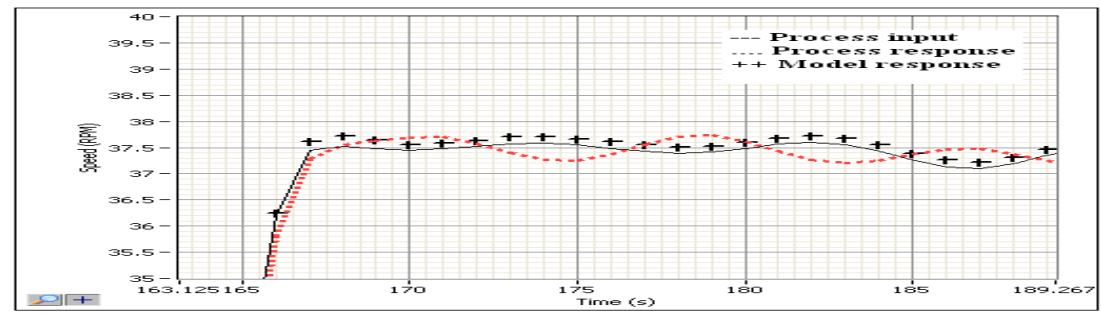

Figure 7: Larger view of vibrations in the process and model response.

However, the very small vibrations in the speed of the process at the output due to the unbalanced mass are noticeable, fig. 7. Here the unbalanced mass is very small nearly 57 gms, which is about $5 \%$ of the mass of the lower rotor representing the drill bit. This mass will represent only a very small bend in the drill string. However, in reality, drill strings when they bend slightly, present more severe vibrations due to the presence of well bore friction and higher mass of the bottom hole assembly. The black box model of the process is identified in a Box Jenkins model format specifically because the Box Jenkins models are good for processes in which disturbances enter late in to the system.

The residual error, figs. 5 and 6 presents us with a model robustness issue which needs to be dealt with. One suggestion is to combine the black box model with a separate model describing effect of the unbalanced mass using analytical principles and larger degrees of freedom Liao et al. [4]. The drilling system prototype concerned here can be seen to be a strictly proper system. In other words the gain tends to zero in the limit as frequency tends to infinity, fig. 8.
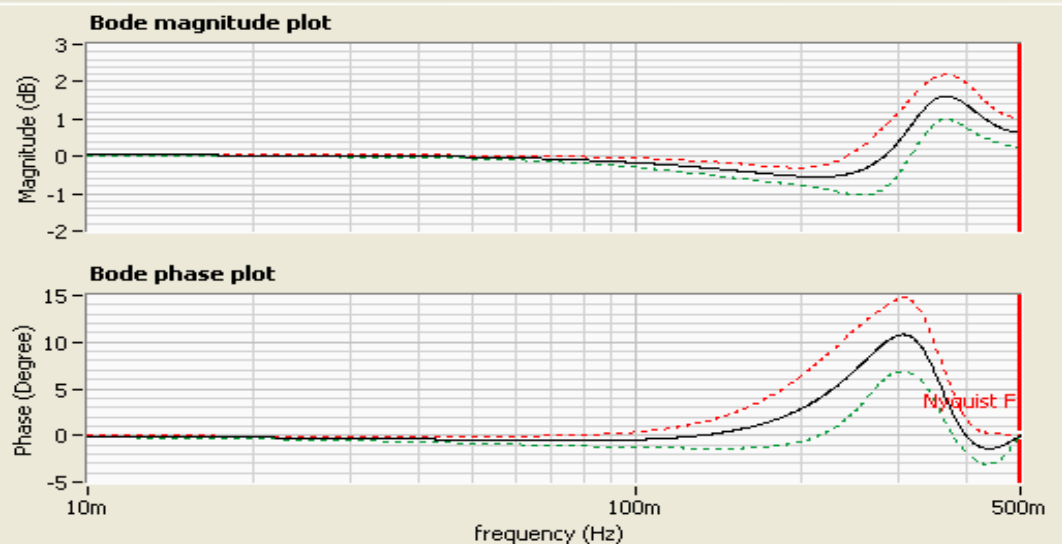

Figure 8: $\quad$ Bode plot of the model with lower (green) and upper (red) bounds.

This can be attributed to the presence of inertia in the system. The model itself will have robustness errors and they need to be analyzed further by looking for RHP poles and zeros, cancellations and analyzing the internal stability of the model. 


\subsection{Modelling error}

Real time systems are nonlinear, time varying, infinite dimensional and very complicated. Modeling error is due to two major reasons.

The model obtained here assumes a third order transfer function; this approximation leads to a simpler model and lack of robustness in the model. This is one of the major sources of modeling error. Assuming that the best model approximation of the plant is $\mathrm{G}_{\mathrm{p}}(\mathrm{s})$;

$$
G_{p}(s)=G(s)+\Delta G(s)
$$

i.e. if

$$
\begin{aligned}
G_{p}(s) & =\sum_{i=1}^{\infty} \frac{N(s)}{D(s)} \\
G(s) & =\sum_{i=1}^{k} \frac{N(s)}{D(s)}
\end{aligned}
$$

and

$$
\Delta G(s)=\sum_{i=k+1}^{\infty} \frac{N(s)}{D(s)}
$$

where $\frac{N(s)}{D(s)}$ is the numerator and denominator of the plant transfer function. $\Delta \mathrm{G}(\mathrm{s})$ is the modeling error, or the difference between the model and the best possible plant model.

Another source of modeling error can be deduced from analyzing the frequency response magnitude; figs. 9 and 10.The frequency response gains are plotted for two different conditions, for small mass unbalance and large mass unbalance. It can be seen that as the frequency increases the size of the resonant peaks tend to decrease after a certain point $\omega$ '. In the frequency response gain plots, the point $\omega$ ' of the drilling system here can be seen to be around $150 \mathrm{~m} \mathrm{~Hz}$ for the two cases studied. This particular frequency $\omega^{\prime}$ is seen to be a constant for a particular system and does not vary with added disturbance, here the unbalanced mass. Hence we can safely assume that for frequencies higher than $\omega$ ' the magnitude of the frequency response will never exceed the gain at that value, i.e;

$$
20 \log _{10}|\Delta G(j w)|=\left\{\begin{array}{l}
=0 ; 0 \leq \omega \leq \omega^{\prime} \\
\leq \rho ; \omega^{\prime}<\omega<\infty
\end{array}\right.
$$

where $\rho$ is the value of gain at $\omega^{\prime}$ and it represents an upper bound on the magnitude of the frequency response of the modeling error.

The second source of error in modeling is from parameter uncertainty. The parameters estimated to obtain the model will have a tolerance associated with their values. Hence at every frequency, the gain and phase response will have an 


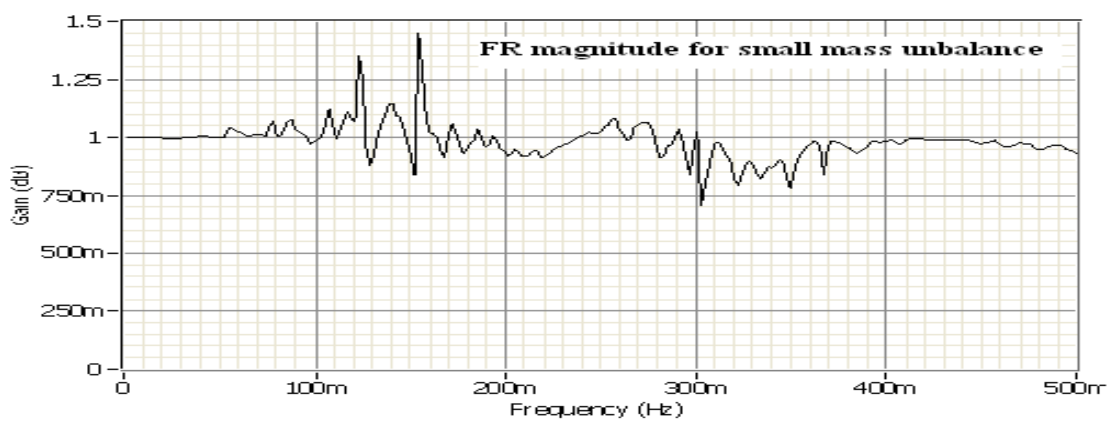

Figure 9: $\quad$ Frequency response magnitude plot for small mass unbalance.

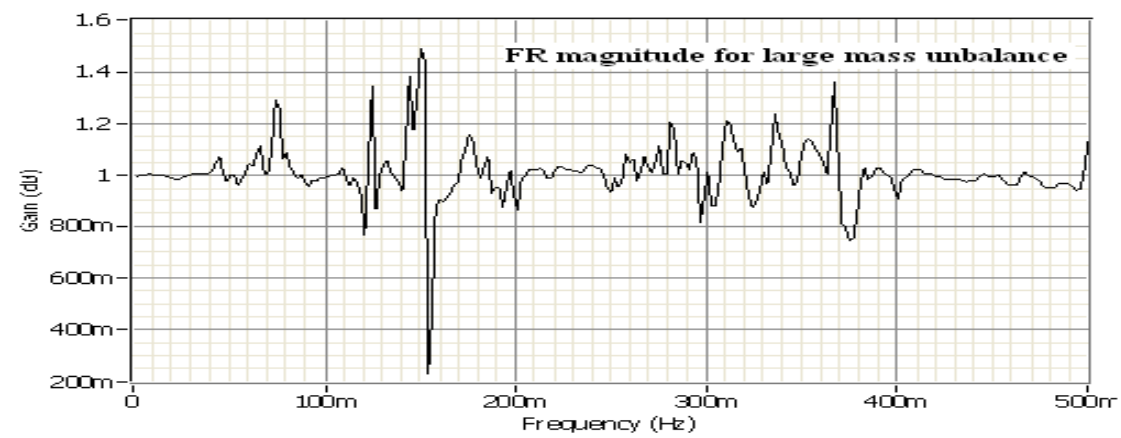

Figure 10: Frequency response magnitude plot for large mass unbalance.

uncertainty associated with its value. These are plotted in the bode plot, fig. 8, with the upper and lower bound of the magnitude and phase curves.

\section{Vibration analysis}

The analytical equation for the lower rotor with the unbalanced mass can be written as Inman [12]:

$$
m \ddot{x}+c \dot{x}+k x=m_{0} e \omega_{r}^{2} \sin \omega_{r} t
$$

where $\mathrm{m}$ is the lower rotor mass, $\mathrm{m}_{0}$ is the unbalanced mass, $\mathrm{c}$ is the damper constant, $\mathrm{k}$ is the spring constant (drill string considered as a spring and damper), $\mathrm{e}$ is the distance of the unbalanced mass from the center axis of rotation of lower rotor, $\omega_{\mathrm{r}}$ is the drilling rotational frequency and $\xi$ is the damping ratio.

The steady state displacement of the lower rotor is

$$
X=\frac{m_{0} e}{m} \frac{r^{2}}{\sqrt{\left(1-r^{2}\right)^{2}+(2 \xi r)^{2}}}
$$

where $r=\frac{\omega_{r}}{\omega_{n}} ; \omega_{\mathrm{n}}$ is the natural frequency. 
At any particular moment $t$, the deflection is:

$$
x_{p}(t)=X \sin \left(\omega_{r} t-\theta\right)
$$

Analyzing the above equations it can be inferred that an increase in the deflection amplitude can be minimized by increasing the damping in the system. It can also be noted that the most vibrations occur when the set up is operated at frequencies near the natural frequency. Hence it is also advisable to operate the set up at frequencies lower or higher than the natural frequency. Sources of model error will be further expanded in forthcoming papers.

For laboratory scale assessment of vibration and related control the Box Jenkins model can be considered appropriate enough due to residual error values not exceeding $200 \mathrm{~m}$ RPM which is $0.4 \%$ of the rotation rate. For control purposes the controller effect is simulated and once an acceptable effect is realized the controller can be interfaced with the drilling setup to analyze experimental response. The under actuation of the lower rotor now presents another difficulty, due to any effect of the controller on the simulated response of the lower rotor will fail to achieve in the experimental set up. The unbalanced mass presents a change in the inertial mass properties of the lower rotor. The vibrations induced can only be minimized by assuring that the drilling is carried out at speeds neglecting the natural frequency, preferably low speeds so that the tangential acceleration is minimized and the drill bit does not cause too much damage to the borehole wall by enlarging it or making wedges in the bore hole and damaging the drill bit and other down hole components.

\section{Summary}

This paper discusses the model of a drill string system representing rotary drilling. The experimental and simulated model responses are plotted and analyzed. The residual error is discussed and the source of the error due to lack of robustness in the model is also studied. Two major reasons for the uncertainty and presence of modeling error are discussed. The source and control of vibration is also discussed. Future work involves study and discussion of robustness issues arising in the model itself, and further expansion of the modeling errors and ways to overcome the errors for a better plant model.

\section{References}

[1] Dykstra, M., Christensen, H., Warren, T., and Azar, J., Drill string component mass imbalance: A major source of drill string vibrations, SPE Drilling and completion, Vol.11, pp.234-241,1996.

[2] Germay, C., van de Wouw, N., Nijmeijer, H., and Sepulchre, R., Nonlinear drill string dynamics analysis, SIAM J. Applied Dynamical systems, Vol. 8, pp.527-553, 2009. 
[3] Leine, van Campen, D.H and Keultjes, W., Stick slip whirl interaction in drill string dynamics, ASME J. of vibration acoustics, Vol.124, pp. 209220, 2002.

[4] Liao. C et al., Reduced order models of drill string dynamics, Paper presented at the Second international energy 2030 conference, UAE, 2008.

[5] Melakhessou, H. et al., A nonlinear well drill string interaction model, J. Vibration acoustics, Vol.125, pp.46 -52, 2003.

[6] Mihajlovioc, N., Van Veggel, A., Van de Wouw, N. and Nijmeijer, H., Friction induced torsional vibrations in an experimental drill string system, Paper presented at the 23rd IASTED international conference on modeling, identification and control, pp.228-233, 2004.

[7] Mihajlovic, N., van Veggel, A., Nan de Wow and Nijmeijer, H., Analysis of friction induced limit cycling in an experimental drill string system, $J$. of Dynamic systems, measurement and control, ASME, Vol.126, pp.709$721,2004$.

[8] Navarro Lopez, E. and Suarez, R., Modeling and Analysis of stick slip behavior in a drill string under dry friction, Congresso Annual De La Amca, pp. 330-336, 2004.

[9] Christoforou, A. and Yigit, A., Dynamic modeling of rotating drill string with borehole interactions, J. sound and vibration, Vol.206, pp.243-260, 1997.

[10] Elsayed, M. A., and Dareing, D. W., Coupling of Longitudinal and Torsional Vibrations in a Single-Mass Model of a Drillstring, Developments in Theoretical and Applied Mechanics, Vol. XVII, University of Arkansas, Fayetteville, AR, pp. 128-139,1994.

[11] Jansen, J.D., Non linear rotor dynamics as applied to oil well drill string vibrations, J. Sound and vibration, Vol.147, pp.115 -135, 1991.

[12] Inman, D.J., Engineering Vibration, Prentice Hall, pp.120-121, 2001. 